\title{
Thermochemistry of electrolyte solutions
}

\author{
Effect of added cosolvent
}

\author{
Henryk Piekarski
}

MEDICTA2011 Conference Special Chapter

(C) The Author(s) 2011. This article is published with open access at Springerlink.com

\begin{abstract}
The results of calorimetric investigations of electrolyte solutions in the mixtures of water, methanol, $\mathrm{N}, \mathrm{N}$-dimethylformamide, and acetonitrile with numerous organic cosolvents are discussed with regard to the intermolecular interactions that occur in the solution. Particular attention is given to answer the questions how and to what extent the properties of the systems examined are modified by the cosolvent added and how much the properties of the cosolvent are revealed in the mixtures with the solvents mentioned above. To this goal, the analysis of the electrolyte dissolution enthalpies, single ionic transfer enthalpies, and enthalpic pair interaction coefficients as well as the preferential solvation (PS) model are applied. The analysis performed shows that in the case of the dissolution enthalpies of simple inorganic electrolytes in waterorganic solvent mixtures, the shape of the dependence of the standard dissolution enthalpy on the mixed solvent composition reflects to a large extent the hydrophobic properties of the organic cosolvent. In the mixtures of methanol with organic cosolvents, the ions are preferentially solvated either by methanol molecules or by molecules of the cosolvent, depending on the properties of the mixed solvent components. The behavior of inorganic salts in the mixtures containing $N, N$-dimethylformamide is mostly influenced by the DMF which is a relatively strongly ion solvating solvent, whereas in acetonitrile mixtures, the thermochemical behavior of electrolyte solutions is influenced to a large extent by the properties of the cosolvent particularly due to the PS of cation by the cosolvent molecules.
\end{abstract}

H. Piekarski $(\bowtie)$

Department of Physical Chemistry, University of Lodz,

90-236 Lodz, Poland

e-mail: kchfpiek@uni.lodz.pl

\section{Introduction}

Most chemical processes occur in solution. For this reason, solutions and liquid mixtures are frequently examined using different experimental methods. In our practice, we apply many different solvents depending on the system examined or our needs and expectation toward its properties. The addition of a cosolvent to the system can modify its properties, sometimes significantly. However, in many cases, it is difficult to predict the direction of these modifications. The same cosolvent added to solutions of given substance in various solvents can cause a different effect depending on the basic solvent. It is so, as aside from the interactions being characteristic of two-component systems, in three-component systems, there also occur solutecosolvent and solvent-cosolvent interactions, and they substantially influence the behavior of the solution. Then a question arises: which of these interactions play a leading role in the solution obtained?

The analysis of interactions in three-component systems is complicated as we have three kinds of molecules interacting with one another in a different way. Therefore, it is convenient to examine solutions of the same solute in a series of binary mixtures containing one chosen solvent and different cosolvents.

Different experimental methods can be applied to examine interactions in solutions. For many reasons, the calorimetry seems to be one of the most useful to this aim as it is the only experimental method allowing direct measurements of the heat effects of various processes and reactions [1]. The total energetic effect of solute-solvent interactions is characterized by the solvation enthalpy, $\Delta_{\text {solv }} H$ that for a solid solute can be calculated from the standard dissolution enthalpy, $\Delta_{\text {sol }} H^{\circ}$ according to the formula: 


$$
\Delta_{\text {solv }} H=\Delta_{\text {sol }} H^{\mathrm{o}}+\Delta H(\text { crystal lattice })
$$

For the description of solutions in binary solvents, the enthalpy of transfer, $\Delta_{\mathrm{tr}} H^{\circ}$, of the solute from an individual solvent (S) to the mixed solvent (M) of various compositions is often used. The knowledge of the enthalpy of crystal lattice formation is not necessary here.

$\Delta_{\mathrm{tr}} H^{\mathrm{o}}=\Delta_{\mathrm{sol}} H^{\mathrm{o}}(\mathrm{M})-\Delta_{\text {sol }} H^{\mathrm{o}}(\mathrm{S})=\Delta_{\text {solv }} H(\mathrm{M})-\Delta_{\text {solv }} H(\mathrm{~S})$

The enthalpy of transfer is a convenient function as it allows one to very easily compare the effect of the mixed solvent composition change on the behavior of different solutes in the same mixtures as well as to compare the effect of the cosolvent added on the properties of solutions of the same solute in different mixed solvents. The frequently observed extreme, inflection or break points on $\Delta_{\mathrm{tr}} H^{\circ}=\mathrm{f}\left(x_{\text {cosolvent }}\right)$ curves are usually interpreted as a result of strong interactions between the solvent and cosolvent molecules leading to changes in the mixture structure with the change in composition and/or preferential solvation (PS), chemical reaction or complex formation in the system. Therefore, the dissolution or transfer enthalpy curves bring much information about interactions in the system. However, in order to get some knowledge about them on a molecular level it is necessary to use the appropriate model of interactions. For analysis of the results of calorimetric investigations on three-component systems, the model of the enthalpic interaction coefficients derived from McMillan-Mayer's theory [2] and Covington's PS concept [3] seems to be very useful.

As is known, the enthalpic pair interaction coefficients can be regarded as a measure of the heat effect (i.e., the enthalpy of interaction) when two solute particles approach each other in dilute solutions. In three-component systems including, solute $\mathrm{X}$, solvent $\mathrm{S}$, cosolvent $\mathrm{Y}$, the enthalpic pair interaction coefficients, $h_{x y}$, illustrate the heat effect of interaction between the solute molecule $\mathrm{X}$ and the cosolvent molecule $\mathrm{Y}$ in the solvent $\mathrm{S}$. As these interactions are solvent mediated, it can be assumed that their values are the sum of two essential energetic effects, namely partial desolvation of interacting species and direct interaction between them [4-7]. The values of pair interaction coefficients in three-component systems can be calculated from the enthalpy of solution or enthalpy of mixing [8].

The PS model proposed by Covington et al. [3] and adapted to the enthalpic effect analysis by Balk and Somsen [9] is based on the simplest version of the thermodynamic theory of PS. The theory analyzes the change in composition of the solvation shell with solvent composition for a given solute $\mathrm{X}$ in a mixture of solvent $\mathrm{S}$ with the cosolvent $\mathrm{Y}$. When the mixture becomes richer in one of its components, the change in the composition of the solvation shell of $\mathrm{X}$ is described by a successive series of $n$ equilibriums, where $n$ is the solvation number:

$\mathrm{X}\left(\mathrm{S}_{n+1-\mathrm{i}} \mathrm{Y}_{i-1}\right)+\mathrm{Y} \quad K_{i} \quad \mathrm{X}\left(\mathrm{S}_{n-i} \mathrm{Y}_{i}\right)+\mathrm{S}(1 \leq i \leq n)$

If $K$ is the equilibrium constant for the process of change in the ionic solvation shell when the mixed solvent composition changes, then $K_{i}=K^{1 / n}$ is the equilibrium constant describing the exchange of one solvent molecule in the ionic solvation shell [9]. When $K<1$, the electrolyte is preferentially solvated by solvent $\mathrm{S}$ while $K>1$ means that the electrolyte is preferentially solvated by cosolvent $\mathrm{Y}$.

In this article, the effect of added cosolvent on the behavior of different solutions is analysed on the base of the results of calorimetric measurements of the solutions of simple electrolytes in mixed solvents carried out for several years mostly in our laboratory. The mixed solvents examined contain: water, methanol $(\mathrm{MeOH}), N, N$-dimethylformamide (DMF), or acetonitrile (AN) as a main solvent and different organic cosolvents. The solvents chosen belong to the most popular ones and they exhibit different properties and structure: water and methanol-both are amphiprotic and hydrogen bonded solvents but they differ from one another in their structure, $N, N$-dimethylformamide is aprotic, proton-acceptor, non-hydrogen bonded solvent whereas $\mathrm{AN}$ is a polar solvent, unable to form H-bonds in "classical" sense neither as proton-donor nor as proton-acceptor. Details about the calorimetric procedure as well as the analysis of the results obtained can be found in the original publications. Here, the results concerning selected systems have been set together and analyzed in order to get an answer to the question posed above.

\section{Analysis of dissolution or transfer enthalpies in binary solvents}

Solutions in water-organic cosolvent mixtures

Let's start from the dissolution enthalpies in water-organic cosolvent mixtures (Fig. 1). As most of these results was published in numerous original and survey papers $[1,4,10$ and references therein], I recall them only shortly. The enthalpies of transfer of simple inorganic electrolytes $(\mathrm{NaCl}, \mathrm{NaI})$ in the mixtures of water with aliphatic alcohols, 2-alkoxyethanols and with aprotic solvents, such as hexamethylphosphotriamide (HMPA), tetrahydrofuran (THF), $N, N$-dimethylacetamide (DMA), 1,2-dimethoxyethane (1,2-DME), and 2-butanone as a function of the of mixed solvent composition exhibit maxima within the range of high water content. These maxima are related to a structure-promotion or a structure-stabilizing effect of added cosolvent on water, mostly due to the hydrophobic hydration of the alkyl 
groups present in the cosolvent molecules [4, 10]. On the other hand, the dissolution enthalpies of the same electrolytes in the mixtures of water with $N, N$-dimetylformamide and dimethylsulfoxide (DMSO) as well as with organic substances which do not contain a non-polar group in their molecules, like formamide and urea, run monotonously within the whole range of the mixed solvent composition $[1,4]$.

The standard solution enthalpies of electrolytes containing ions with alkyl or aryl groups exhibit maxima in the water rich region in all the systems examined thus far $[4,10,11]$. Their height and position depend on the number and size of alkyl or aryl groups in the organic ion. It suggests that the presence of these maxima is also connected with hydrophobic effects and probably can be attributed to competition between organic cosolvent and organic ions for the water molecules needed to form hydration shells [10].

It was concluded that the shape of the $\Delta_{\mathrm{sol}} H_{\mathrm{m}}{ }^{\circ}=$ $f(x)$ curves in water-organic cosolvent mixtures, at least in the range of high water content, depends on the hydration susceptibility of the organic cosolvent molecule, understood as a resultant of the hydrophobic effect of non-polar groups and the effect of hydrophilic interactions of functional polar groups present in the molecule. In some cases the latter interactions are strong enough to overcome the hydrophobic hydration effects and then the maximum of the dissolution enthalpy of inorganic electrolytes is relatively small or even it is not observed [1].

In order to get additional arguments to confirm the above conclusions the enthalpic pair interaction coefficients were determined. To facilitate the analysis, the enthalpic pair interaction coefficients $h_{x y}$ for a series of pairs consisting one, selected solute $\mathrm{X}$ (for instance: $\mathrm{NaI}$ ) and different cosolvents $\mathrm{Y}$ in solutions were considered. In this way the variations of the $h_{x y}$ values depended on the differences in the solvation effects of the substance $\mathrm{Y}$ and in the effects of direct interaction between $X$ and Y. Such approach made it possible to obtain, indirectly, some information about the interactions between the solvent and cosolvents [1,4].

The enthalpic pair interaction coefficients for the pairs of NaI with different non-electrolytes (used as cosolvents) in water have different values both positive and negative, depending on the kind of the non-electrolyte (Table 1). The positive values, characteristic of alcohols, and other substances whose molecules contain large apolar groups (e.g., THF, HMPA, 1,2-DME) prove the domination of the endothermic effects of desolvation over the effects of direct interactions between the ions and the non-electrolyte molecule. On the contrary, the negative $h_{x y}$ values observed for non-electrolytes with strong polar group in their molecule like: formamide (FA), acetamide (AA), urea, DMSO, reflect the advantage of the exothermic
Table 1 Enthalpic pair interaction coefficients, $h_{x y} / \mathrm{J} \mathrm{kg} \mathrm{mol}^{-2}$ and "linear" $h_{x y}{ }^{*}$ and "specific" $h_{x y}^{E}$ contributions to $h_{x y}$ for NaI-nonelectrolyte pairs in water and NaI-alkyl groups interaction parameters, $h_{x \text {-alkyl }}$ calculated from functional group parameters

\begin{tabular}{lrrrr}
\hline Non-electrolyte (Y) & \multicolumn{1}{c}{$h_{x y}$} & \multicolumn{1}{c}{$h_{x y}{ }^{*}$} & \multicolumn{1}{l}{$h_{x y}^{E}$} & $h_{x \text {-alkyl }}$ \\
\hline MeOH & 314 & -220 & 534 & 445 \\
EtOH & 596 & -154 & 750 & 742 \\
PrOH & 780 & -156 & 936 & 1040 \\
i-PrOH & 1018 & -184 & 1202 & 1040 \\
i-BuOH & 1200 & -184 & 1384 & 1337 \\
s-BuOH & 1286 & & & \\
t-BuOH & 1440 & & & \\
1,2-ED & 178 & & & \\
1,2-PD & 381 & & & \\
1,2-BD & 518 & & & \\
2-ME & 194 & -466 & 660 & 1040 \\
1,2-DME & 210 & & & \\
Acetone & -92 & -328 & 236 & 894 \\
2-Butanone & 140 & & & \\
Urea & -524 & & & \\
FA & -696 & -213 & -483 & \\
DMF & -350 & -440 & 90 & 891 \\
DMA & -124 & & & \\
AN & -494 & -190 & -304 & 297 \\
DMSO & -628 & -364 & -264 & \\
THF & 344 & & & \\
MPA & 564 & & & \\
\hline
\end{tabular}

effect of direct interactions. It was found that the enthalpic pair interaction coefficients for the NaI-non-electrolyte pairs in water were linearly correlated with functions describing some properties of the non-electrolytes, such as molecular polarizability $(\alpha)$, or DimrothReichardt polarity parameter $\left(E_{\mathrm{T}}\right)$ [12]. However, these correlations hold only for groups of related compounds. An interesting correlation, which included most of the systems investigated so far, was obtained between the $h_{x y}$ for NaI-non-electrolyte pairs in water, and the heat capacity of interactions, $C_{p}$ (int) between the non-electrolyte $\mathrm{Y}$ and solvent water [7] (Fig. 2). Similar correlations were obtained when the $\mathrm{NaI}$ was replaced by $\mathrm{NaCl}$, or by polar non-electrolyte such as urea [7]. From the analysis of the dependences mentioned above it was concluded that in aqueous solutions for a given solute the hydration effects of the cosolvent molecules made a leading contribution to the observed variation of the $h_{x y}$ values in water. Other possible contributions do not decisively influence the $h_{x y}$ variation. However, within a group of substances having similar molecular structure and properties (alcohols, aprotic compounds) the effects of donoracceptor $\left(E_{\mathrm{T}}\right)$ or electrostatic $(\alpha)$ interactions play some 
diversifying role $[1,4]$. This conclusion can be confirmed when we split the $h_{x y}$ coefficients into "linear" $h_{x y} *$ and "specific" or "excess" $h_{x y}^{\mathrm{E}}$ contributions: $h_{x y}=h_{x y}{ }^{*}+h_{x y}^{E}$ [13]. The former one, $h_{x y}{ }^{*}=\left(\mathrm{M}_{\mathrm{S}} / 2\right) \Delta_{\mathrm{tr}} H^{0}(\mathrm{~S} \rightarrow \mathrm{Y})$ derived on the basis of the assumption about the linearity of the dissolution enthalpy of the electrolyte in the mixed solvents illustrates the effect of gradual changes in the solute solvation shell structure. The latter, $h_{x y}^{E}$, illustrates the effect arising from the 'specific' interactions in the system under investigation (e.g., PS). The results of calculations are presented in Table 1. As it can be seen, the main contribution to the pairs of NaI with DMSO and with DMF come from the 'linear' term, which indicates that in these systems, the $h_{x y}$ coefficients result from a simple replacement of water molecules by DMF or DMSO, respectively, in the solvation shell of the ions. For most of the other discussed pairs in water, a significant input from 'specific' interactions to $h_{x y}$ is observed. These positive values of $h_{x y}^{E}$ reflect mainly the destruction of the hydrophobic shells of the apolar groups in the cosolvent molecule. This conclusion is supported by the effect of interactions between the $\mathrm{NaI}$ and the alkyl groups, calculated from the group interacting contributions determined on the base of Savage and Wood's group additivity model [14].

Summarizing, one can say that in the case of the solutions of simple inorganic electrolytes like NaI, in waterorganic cosolvent mixtures, the diversification of the ion solvation effect in the range of high water content is

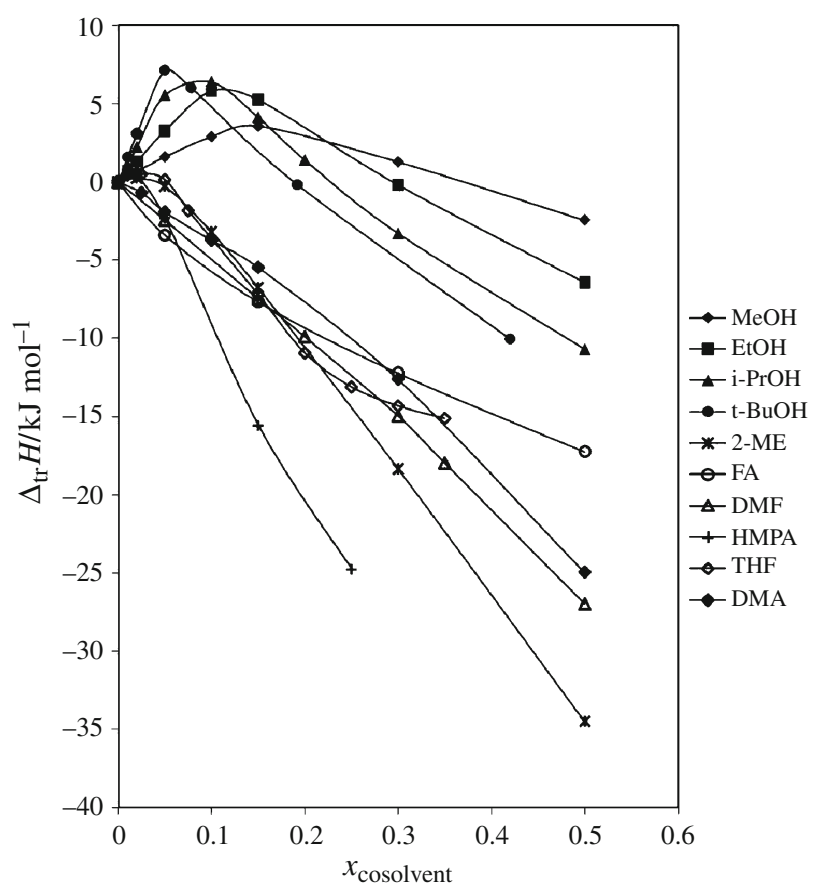

Fig. 1 Enthalpies of transfer of $\mathrm{NaI}$ from water to water-cosolvent mixtures at $298.15 \mathrm{~K}$ dominated by water-organic cosolvent interactions, i.e., by organic cosolvent hydration.

Solutions in methanol-cosolvent mixtures

The dissolution enthalpies of $\mathrm{NaI}$ in methanol-cosolvent mixtures exhibit various shapes (Fig. 3). In the mixtures of methanol with ethanol, $n$-propanol and iso-propanol, the addition of the cosolvent brings about a slow monotonous growth of the NaI dissolution enthalpy with no maxima [15]. 1,2-diols as cosolvents behave similarly in the range of high methanol content [16]. The absence of the maxima of the $\Delta_{\text {sol }} H^{\circ}$ in these mixtures within the methanol-rich range seems to point to the lack of ordering structure effect or the phenomenon analogous to the hydrophobic hydration (i.e., solvophobic solvation) [10]. The curves of the solution enthalpy of $\mathrm{NaI}$ in the mixtures of methanol with three aprotic solvents having similar electric permittivity (DMF, AN, NM) have different shapes [17]. It follows from the analysis that in these almost isodielectric mixtures, the ioncosolvent interactions connected with the donor-acceptor properties of the mixed solvent components are more important than their electric permittivity. On the other hands, in the mixtures of methanol and propylene carbonate the dissolution enthalpy of $\mathrm{NaI}$ does not substantially change its value within the wide range of the $\mathrm{MeOH}$ content [18]. From the point of view of this article topic, it is important that in the range of high methanol content in the mixed solvents, both the growth and decrease in the dissolution enthalpies of $\mathrm{NaI}$ depending on the kind of the cosolvent are observed. The same concerns the transfer enthalpies of electrolytes with organic ions [18-23] as well as the single ionic transfer enthalpies [18-23]. Therefore,

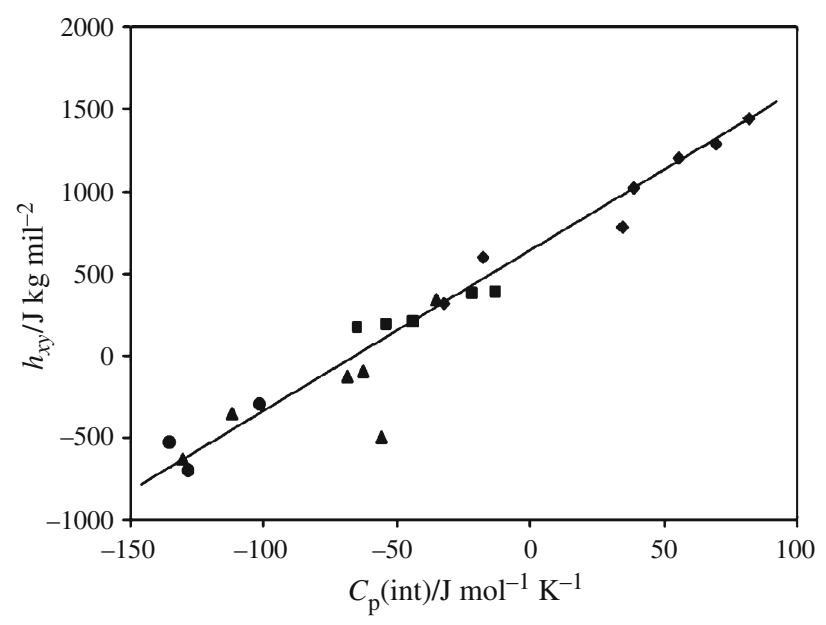

Fig. 2 Enthalpic pair interaction coefficients, $h_{x y}$ for NaI-nonelectrolyte pairs in water as a function of $C_{\mathrm{p}}$ (int) of the nonelectrolyte; alcohols (filled diamond), bifunctional compounds (filled square), aprotic compounds (filled triangle), hydrophilic compounds (filled circle) 
Table 2 Enthalpic interaction coefficients, $h_{\mathrm{xy}} / \mathrm{J} \mathrm{kg} \mathrm{mol}{ }^{-2}$ for NaInon-electrolyte pairs in methanol

\begin{tabular}{lr}
\hline Non-electrolyte (Y) & \multicolumn{1}{c}{$h_{x y}$} \\
\hline EtOH & 31 \\
PrOH & 23 \\
i-PrOH & 26 \\
$1,2-\mathrm{ED}$ & $\sim 0$ \\
$1,2-\mathrm{PD}$ & 82 \\
$1,2-\mathrm{BD}$ & 153 \\
$1,2-\mathrm{PeD}$ & 343 \\
AN & -335 \\
DMF & -950 \\
PC & -20 \\
NM & 40 \\
\hline
\end{tabular}

Table 3 Preferential solvation fit parameters of $\mathrm{NaI}$ in methanol + cosolvent mixtures

\begin{tabular}{lcl}
\hline Cosolvent $(\mathrm{Y})$ & $K^{1 / n}$ & $n$ \\
\hline DMF & 15.4 & 1.2 \\
AN & 0.07 & 3.4 \\
PC & 0.08 & 2.1 \\
EtOH & 1.00 & - \\
\hline
\end{tabular}

the addition of a cosolvent to methanol modifies its behavior as the solvent for simple electrolytes, which is reflected by different shapes of the transfer enthalpy curves from methanol to methanol-cosolvent mixtures in the range of high methanol content both for the electrolytes and single ions. It means that the solvation properties of the cosolvent toward the solute are preserved to some extent in methanol mixtures.

The values of the enthalpic interaction coefficients for $\mathrm{NaI}-$ cosolvent pairs in methanol have different values, both positive and negative, depending on the cosolvent properties [16] (Table 2). The correlations of the $h_{\mathrm{xy}}$ (NaI-cosolvent) coefficients that are characteristic of aqueous solutions were not observed here. One can suppose that in methanol solution, the effects of ion-cosolvent molecule interactions are comparable with the ion-methanol interactions and possibly the effects of selective solvation of the ions diversify much stronger the $h_{x y}$ values than it happen in aqueous solutions. In order to verify this suggestion, it is possible to use the PS model proposed by Covington et al. mentioned earlier [3, 9]. The PS parameters $K^{1 / n}$ and $n$ for NaI in some of the methanol mixtures examined are given in Table 3. The values of the reaction equilibrium constants in the mixtures of $\mathrm{MeOH}$ and DMF are higher than unity $(K>1)$, which indicates PS by the cosolvent in these mixtures. However, in the mixtures of $\mathrm{MeOH}$ and $\mathrm{PC}$ as well as $\mathrm{MeOH}$ and $\mathrm{AN}$, the reaction

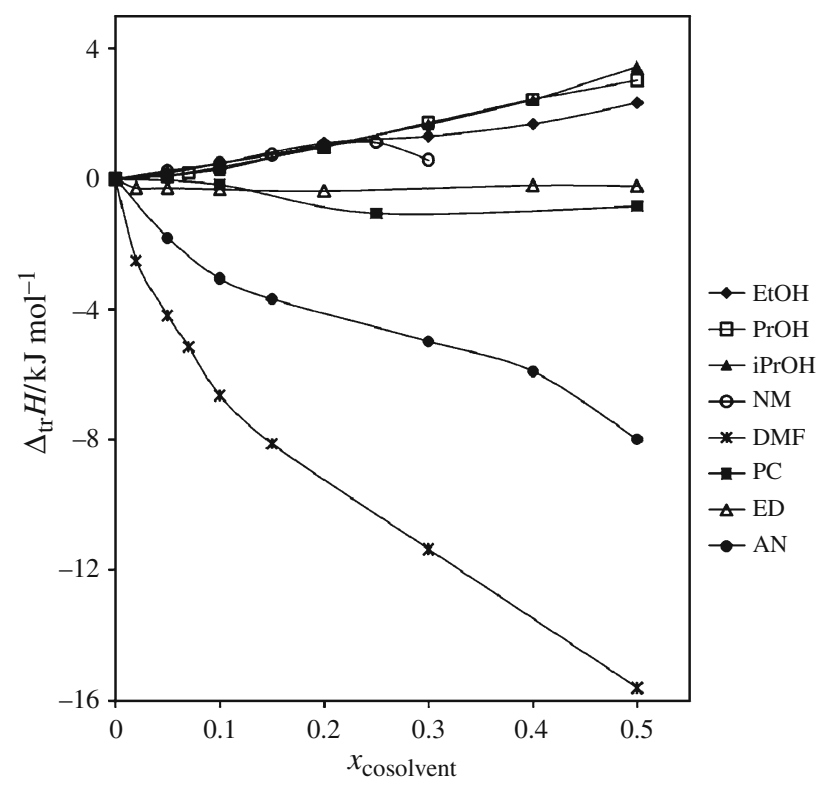

Fig. 3 Enthalpies of transfer of NaI from methanol to methanolcosolvent mixtures at $298.15 \mathrm{~K}$

equilibrium constants are lower than one $(K<1)$, which means that electrolytes are preferentially solvated by methanol. In the mixture of $\mathrm{MeOH}$ and $\mathrm{EtOH}$ the equilibrium constant is equal to unity $(K=1)$, which shows that in this system the NaI solvation shells change gradually with the change in the mixed solvent composition from typical of $\mathrm{MeOH}$ to typical of $\mathrm{EtOH}$.

To sum up the above, the competition between the ionmethanol and ion-cosolvent interactions in $\mathrm{NaI}$ electrolyte solutions in methanol-cosolvent mixtures influences the behavior of the system already in the range of low cosolvent content. In some cases, it leads to the PS of the solute.

Solutions in $N, N$-dimethylformamide-cosolvent mixtures

The enthalpies of transfer of $\mathrm{NaI}$ in all the mixtures of $\mathrm{N}, \mathrm{N}$-dimethylformamide and organic cosolvents examined are positive and they increase with increasing cosolvent content in the mixture [13, 24, 25] (Fig. 4). In this respect, the enthalpies under discussion behave differently than those in water-cosolvent and methanol-cosolvent mixtures, where different shapes of the enthalpy of transfer curves were observed within the range of low cosolvent content. The slope of the transfer enthalpy function depends on the kind of the cosolvent and it increases in the following order: aprotic cosolvents $<$ alcohols and secondary amides $<$ primary amides (and urea). It is interesting to mention that the single ionic transfer enthalpies for inorganic cations behave in the same manner [21]. They increase along with the increase in the cosolvent content. 


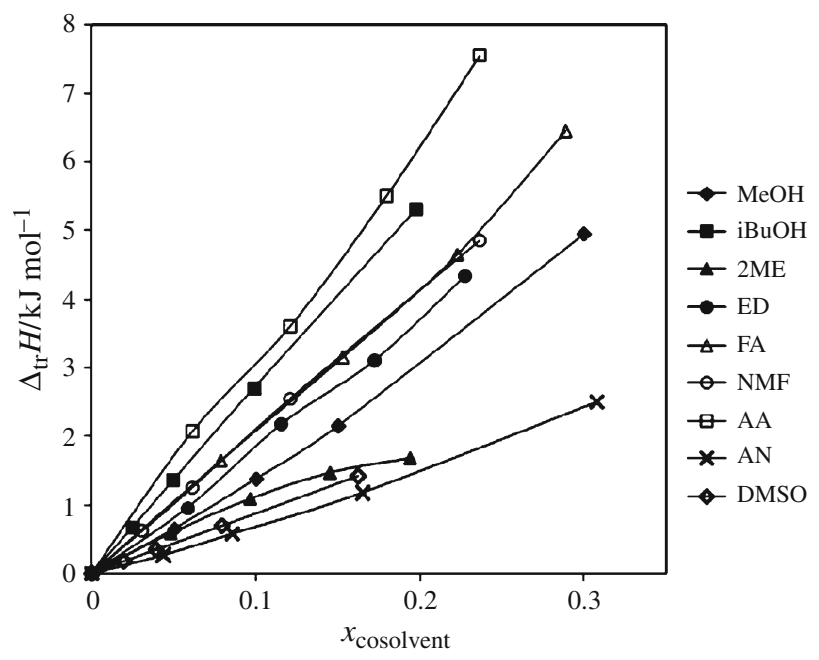

Fig. 4 Enthalpies of transfer of $\mathrm{NaI}$ from $N, N$-dimethylformamide (DMF) to DMF-cosolvent mixtures at $298.15 \mathrm{~K}$

Table 4 Enthalpic pair interaction coefficients, $h_{x y} / \mathrm{J} \mathrm{kg} \mathrm{mol}^{-2}$ and "linear" $h_{x y}{ }^{*}$ and "specific" $h_{x y}{ }^{E}$ contributions to $h_{x y}$ for NaI-nonelectrolyte pairs in $N, N$-dimethylformamide

\begin{tabular}{lrrr}
\hline Non-electrolyte (Y) & \multicolumn{1}{c}{$h_{x y}$} & $h_{x y}{ }^{*}$ & $h_{x y}{ }^{E}$ \\
\hline $\mathrm{MeOH}$ & 460 & 894 & -434 \\
$\mathrm{PrOH}$ & 860 & 1146 & -286 \\
i-PrOH & 960 & 1040 & -80 \\
i-BuOH & 1000 & 1032 & -32 \\
$\mathrm{NMF}$ & 760 & 800 & -40 \\
DMSO & 270 & 300 & -30 \\
Acetone & 420 & 448 & -28 \\
THF & 500 & & \\
AN & 240 & 1010 & -770 \\
\hline
\end{tabular}

Also the salts with organic cation exhibit similar properties $[11,21,26]$. On the other hand, the transfer enthalpies of polar non-electrolyte, such as urea in the same mixtures behave in reverse. They are negative and decrease with increasing cosolvent content within the range of low cosolvent content [27]. As follows from the detailed discussion, the observed shape of the transfer (and dissolution) enthalpies of the electrolytes $\left(\mathrm{NaI}, \mathrm{Bu}_{4} \mathrm{NBr}\right)$ in examined DMF-cosolvent mixtures reflect, to a large extent, the effect of energetically unfavorable replacement of DMF molecules by the cosolvent ones in the solvation region of the electrolyte $[13,24,25]$. This conclusion can be confirmed by the analysis of the enthalpic pair interaction coefficients for NaI-cosolvent pairs in DMF [13, 24, 25]. Their values are positive in all DMF solutions examined thus far (Table 4). Moreover, the enthalpic pair interaction coefficients in DMF do not exhibit the correlations observed in water. The lack of correlation with the heat capacity of the non-electrolyte solvation indicates that the energetic effect of changes in the solvent structure in the vicinity of the dissolved particles leads to a variation in $h_{x y}$ values in DMF to a lesser extent than that, which occurs in water as a solvent. It is so as DMF is less structured than water in its liquid state. Therefore, the direct interactions between electrolyte and non-electrolyte, connected with the energetically unfavorable replacement of DMF molecules by non-electrolyte molecules in solvation region of the ions, seem to play a very important role in this system. In order to throw some additional light on this problem the 'linear' and 'specific' contributions to the $h_{x y}$ coefficients have been calculated [13]. The results obtained (Table 4) indicate that the 'linear' contribution, $h_{x y}{ }^{*}$, plays significant role in variation of the $h_{x y}$ values. The values of $h_{x y} *$ in DMF for the pairs of NaI with NMF ACT, DMSO i-PrOH, and i-BuOH are very close to $h_{x y}$, which means that the 'specific' contributions are negligible, $h_{x y}^{E} \approx 0$ (within the error limits). Therefore, the positive values of $h_{x y}$ observed reflect, indeed, the energetically unfavorable replacement of DMF molecules by the cosolvent in the solvation shell of the electrolyte in these systems. In the other systems (the lower alcohols, 2-ME, AN) examined, we observe a relatively large negative 'specific' contribution. It is possible that the strong solvation of both the NaI and cosolvent molecules in DMF makes the effect of partial desolvation of the interacting species less differentiated than in water and in methanol.

It arises from the discussion presented that $N, N$ dimethylformamide relatively weakly differentiates the properties of cosolvents. A decisive part in the behavior of electrolyte solutions within the DMF-rich region in the system is played by the properties of DMF as solvent that relatively strongly solvates ions.

\section{Solutions in AN-organic cosolvent mixtures}

The shape of the transfer enthalpies of simple inorganic electrolytes from pure $\mathrm{AN}$ to examined $\mathrm{AN}-$ cosolvent mixtures (including DMA, DMF, DMSO, 2-ME, $\mathrm{MeOH}$ as cosolvents) is different from that observed in systems presented earlier. It is characterized by a sharp drop within the range of high AN content in the mixed solvent, and then an almost linear course to the value in pure cosolvent $[17,20,22,23,28,29]$. The transfer enthalpy curves in the mixtures of AN and PC run almost linearly within the whole range of the mixed solvent composition [28] (Fig. 5). The characteristic shape of the dissolution enthalpy curves observed seems to be unconnected with the rapid change in the mixed solvent structure. The numerous thermodynamic functions characterizing the AN mixtures examined, which are very sensitive to any changes that occur in the system, have their values almost independent of the mixture composition [30-32]. The rapid decrease in the enthalpy of solvation (or transfer) due to the addition of 


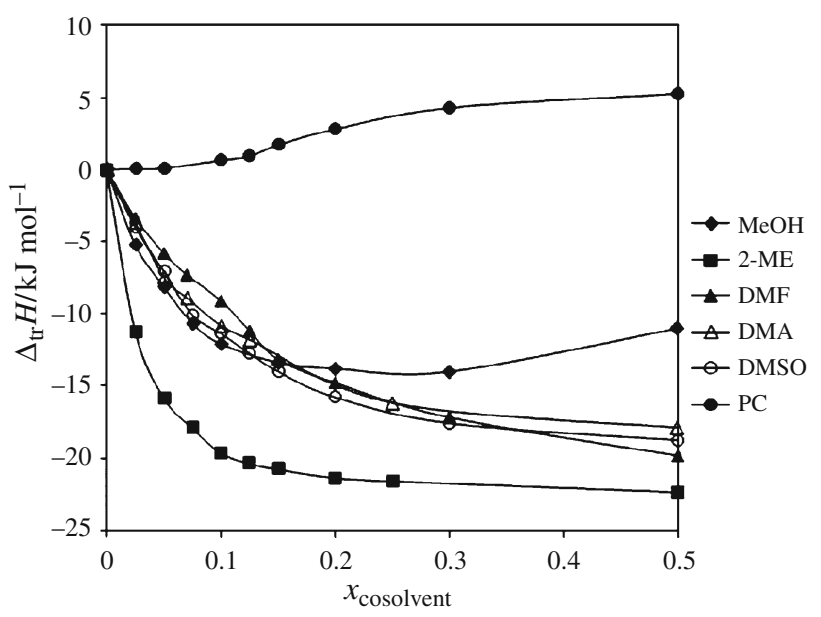

Fig. 5 Enthalpies of transfer of NaI from acetonitrile to acetonitrilecosolvent mixtures at $298.15 \mathrm{~K}$

Table 5 Preferential solvation fit parameters of $\mathrm{NaI}$ and $\mathrm{Na}^{+}$in acetonitrile + cosolvent mixtures

\begin{tabular}{lrrrrr}
\hline Cosolvent (Y) & \multicolumn{2}{c}{$\mathrm{NaI}$} & & \multicolumn{2}{c}{$\mathrm{Na}^{+}$} \\
\cline { 2 - 3 } & \multicolumn{1}{c}{$K^{1 / n}$} & $n$ & & \multicolumn{1}{c}{$K^{1 / n}$} & \multicolumn{1}{c}{$n$} \\
\hline MeOH & 14.6 & 3.4 & 6.4 & 5.2 \\
2-ME & 46.4 & 2.3 & 31.8 & 1.8 \\
DMA & 8.6 & 3.3 & & \\
DMF & 11.6 & 3.0 & & 9.6 & 3.5 \\
DMSO & 9.1 & 4.2 & & \\
PC & 1.3 & & & \\
\hline
\end{tabular}

cosolvent to the solution can be attributed to the replacement of AN molecules within the solvation shell of electrolyte by stronger solvating molecules of cosolvent. The composition of the ionic solvation sphere in most of the systems discussed becomes stable in the systems containing about $70 \mathrm{~mol} \%$ of AN. The mixture of AN and PC is an exception, probably due to the comparably weak capacity for the solvation of ions by both solvents [28-30].

The analysis of the single ion transfer enthalpies revealed that the observed changes in $\Delta_{\mathrm{sol}} H^{\circ}$ of sodium salts in the range of high AN content are due to the PS of the sodium cation [22, 28, 29]. To confirm the above conclusion, the model of PS was used. The values of the reaction equilibrium constants $K^{1 / n}$ for $\mathrm{NaI}$ and $\mathrm{Na}^{+}$in almost all of the examined mixtures of AN are higher than unity $(K>1)$, which indicates preferential ionic solvation by cosolvent in the mixtures of AN with these cosolvents [29]. The only exception is the AN + PC mixture for which the equilibrium constant for $\mathrm{NaI}$ solvation amounts to 1.3 (Table 5). It confirms the earlier conclusion that in the latter system the structure of ionic solvation shells changes gradually along with the change in the mixed solvent composition.
Table 6 Enthalpic pair interaction coefficients, $h_{x y} / \mathrm{J} \mathrm{kg} \mathrm{mol}{ }^{-2}$ and "linear" $h_{x y}{ }^{*}$ and "specific" $h_{x y}{ }^{E}$ contributions to $h_{x y}$ for NaI-nonelectrolyte pairs in acetonitrile

\begin{tabular}{lrrr}
\hline Non-electrolyte (Y) & \multicolumn{1}{l}{$h_{x y}$} & \multicolumn{1}{l}{$h_{x y}{ }^{*}$} & \multicolumn{1}{l}{$h_{x y}{ }^{E}$} \\
\hline DMF & -2925 & -283 & -2642 \\
DMA & -3337 & -235 & -3102 \\
DMSO & -3050 & -206 & -2844 \\
PC & 3 & 80 & -77 \\
2-ME & -5493 & -267 & -5226 \\
$\mathrm{MeOH}$ & -2370 & -31 & -2339 \\
\hline
\end{tabular}

The values of the enthalpic pair interaction coefficients for $\mathrm{NaI}-$ non-electrolyte pairs in $\mathrm{AN}$, are negative for almost all the systems examined [28, 29]. And again the system containing PC, whose $h_{x y}$ value is close to zero is the only exception (Table 6). The highly negative values of the "specific", $h_{x y}^{E}$, contributions also show us that the NaIcosolvent molecule interactions leading to the replacement of the solvent molecules by the cosolvent ones in the NaI solvation shell are dominating in the AN solution. The interpretation of the behavior of NaI-acetonitrile-cosolvent systems presented above is additionally proved by linear correlation of the enthalpic pair interaction coefficients in an for the pairs containing NaI and DMF, DMA, DMSO or 2-ME with the values of $\ln K^{1 / n}$ derived from Covington's PS model for the same systems [28, 29].

Then, the observed behavior of the system mainly reflects the properties of cosolvent, particularly the cationcosolvent interactions. A dominating part in the behavior of electrolyte solutions within the AN-rich region is played by the PS of electrolyte, especially cation by cosolvent and relatively weak interaction between the mixed solvent components [29].

\section{Summary and final remarks}

In this article, the effect of added cosolvent on the properties of four selected liquids: water, methanol, $\mathrm{N}, \mathrm{N}$ dimethylformamide, and AN, used as solvents for simple electrolytes, has been analyzed. The results obtained, which concern the particular solvents, together with some assessment of the reasons for the behavior of the systems examined are compared below. That scheme makes it possible to choose an optimal or at least advantageous solvent for studies depending on the research subject and what kind of interactions we are interested in. Are the leveling properties of DMF more important for us than the diversifying effect of methanol or relatively neutral behavior of AN? Which of these solvents is better as a reference one? 
It is also very characteristic that for the electrolyte solutions in the three organic solvents $(\mathrm{MeOH}, \mathrm{DMF}, \mathrm{AN})$ examined, the solvation effects predominantly influence the properties of the system observed calorimetrically. However, in aqueous solutions of single, non-reacting electrolytes, the water-organic cosolvent interactions decisively influence the behavior of the system.

\section{Solvation properties of the binary solvents}

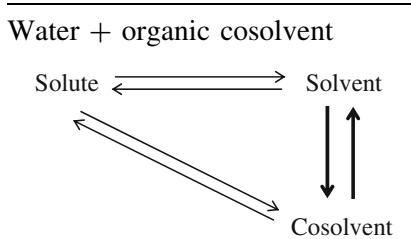

The effect of ion solvation within the water-rich region is dominated by the hydration of the organic cosolvent-rich range, its influence on the solvation of ions becomes dominating or at least considerable.

$\mathrm{DMF}+$ organic cosolvent

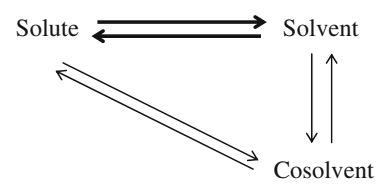

DMF relatively weakly differentiates the properties of the cosolvents. A decisive part in the behavior of electrolyte solutions within the DMF-rich region in the system is played by the properties of DMF as solvent that relatively strongly solvates ions. organic cosolvent. Only within

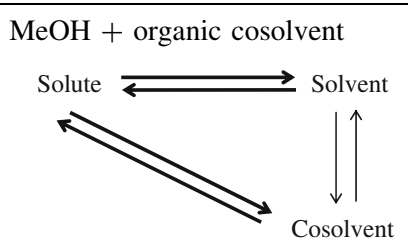

The addition of cosolvent to methanol modifies the properties of methanol as a solvent for electrolytes, already with the addition of its first portion. Depending on the kind of the cosolvent, the PS of ions is observed.

$\mathrm{AN}+$ organic cosolvent

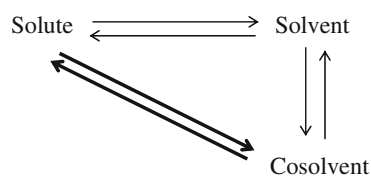

The behavior of the system reflects mainly the properties of cosolvent, especially the cation-cosolvent interactions.

A dominating role in the behavior of electrolyte solutions within the AN-rich region is played by the PS of electrolyte (cation) by the cosolvent. by methanol or by the cosolvent

And, the last but not least, the calorimetry, also in this case, showed its usefulness as a tool for the examination of solution properties.

Open Access This article is distributed under the terms of the Creative Commons Attribution Noncommercial License which permits any noncommercial use, distribution, and reproduction in any medium, provided the original author(s) and source are credited.

\section{References}

1. Piekarski H. Calorimetry: an important tool in solution chemistry. Thermochim Acta. 2004;420(1-2 Spec. Iss):13-8.

2. McMillan W G Jr, Mayer E. The statistical thermodynamics of multicomponent systems. J Chem Phys. 1945;13:276-305.
3. Covington AK, Thain JM. Nuclear magnetic resonance studies of preferential solvation. Part 4: Thermodynamic treatment involving non-statistical distribution of solvated species. J Chem Soc Faraday Trans 1. 1974;70:1879-87.

4. Piekarski H. Application of calorimetric methods to investigations of interactions in solutions. Pure Appl Chem. 1999;71: 1275-83.

5. Piekarski $\mathrm{H}$. Dissolution enthalpy of $\mathrm{NaCl}$ in nonaqueous nonelectrolyte solutions at $298.15 \mathrm{~K}$. Analysis of electrolyte-nonelectrolyte enthalpic pair interaction coefficients in aqueous solution. Can J Chem. 1986;64:2127-31.

6. Piekarski H, Somsen G. Enthalpies of solution of urea in wateralkanol mixtures and the enthalpic pair interaction coefficients of urea and several nonelectrolytes in water. Can J Chem. 1986; 64:1721-4.

7. Piekarski H, Tkaczyk M. Effect of non-electrolyte properties on the enthalpic interaction coefficients for $\mathrm{NaCl} / \mathrm{NaI}$-non-electrolyte pairs in water. Dissolution enthalpies of $\mathrm{NaCl}$ and $\mathrm{NaI}$ in aqueous solutions of butan-2-one and 1,2-dimethoxyethane at $25^{\circ}$ C. J Chem Soc Faraday Trans. 1991;87:3661-6.

8. Desnoyers JE, Perron G, Avedikian L, Morel J-P. Enthalpies of the urea-tert-butanol-water system at $25^{\circ} \mathrm{C}$. J Solution Chem. 1976;5:631-44.

9. Balk RW, Somsen G. Preferential solvation and hydrophobic hydration of polyols in mixtures of water and N, N-dimethylformamide. J Phys Chem. 1985;89:5093-7.

10. Taniewska-Osińska S, Electrolytes in binary solvents: an experimental approach. Chem Soc Rev. 1993; 205-12.

11. Somsen G. Solution calorimetry of alkyl substituted ammonium bromides in aqueous and nonaqueous solvents. Pure Appl Chem. 1993;65:983-90.

12. Piekarski H. Dissolution enthalpies of $\mathrm{NaI}$ in binary mixtures of water with aliphatic alcohols and methylcellosolve at $298.15 \mathrm{~K}$. Enthalpic pair interaction coefficients of NaI-nonelectrolyte in water solution. Can J Chem. 1983;61:2203-8.

13. Piekarski H. Enthalpic pair interaction coefficients of NaI-nonelectrolyte pairs in DMF solution at $25^{\circ} \mathrm{C}$. J Chem Soc Faraday Trans 1. 1988;84:591-9.

14. Savage JJ, Wood RH. Enthalpy of dilution of aqueous mixtures of amides, sugars, urea, ethylene glycol and pentaerythritol at $25^{\circ} \mathrm{C}$ : enthalpy of interaction of the hydrocarbon, amide and hydroxyl functional groups in dilute aqueous solutions. J Solution Chem. 1976;5:733-50.

15. Taniewska-Osińska S, Piekarska A. Calorimetric investigation of $\mathrm{NaI}-$ alcohol interactions in alcohol + alcohol mixtures. J Chem Soc Faraday Trans 1. 1985;81:1913-21.

16. Piekarski H, Pietrzak A, Enthalpic interaction coefficients of $\mathrm{NaI}$ - alkanediol pairs in methanol and in water. J Therm Anal Calorim. (2011) doi:10.1007/s10973-011-1603-9

17. Piekarska A, Piekarski H, Taniewska-Osińska S. Calorimetric studies of NaI solutions in binary organic isodielectric mixtures. J Chem Soc Faraday Trans 1. 1986;82:513-20.

18. Piekarska A. Ion solvation in methanol-organic cosolvent mixtures Part 5. Enthalpies of transfer of inorganic ions in mixtures of methanol and propylene carbonate at $298.15 \mathrm{~K}$. Thermochim Acta. 1994;244:61-7.

19. Kondo Y, Yuki K, Yoshida T, Tokura N. Nucleophilic substitution in binary mixed solvents: kinetics and transfer enthalpies of anions in the mixed solvents methanol + propylene carbonate and methanol $+N$-methyl-2-pyrrolidone. J Chem Soc Faraday Trans 1. 1980;76:812-24.

20. Kondo Y, Ittoh M, Kusabayashi S. Reaction of ethyl iodide with bromide ion. Kinetics, transfer enthalpies of anions and extended Brönsted relationships in acetonitrile + methanol and $\mathrm{N}$, $\mathrm{N}$-dimethylacetamide + methanol mixtures. J Chem Soc Faraday Trans 1. 1982;78:2793-806. 
21. Taniewska-Osińska S, Piekarska A. Single-ion transfer enthalpies for $\mathrm{Ph}_{4} \mathrm{P}^{+}=\mathrm{BPh}_{4}{ }^{-}, \mathrm{Na}^{+}$and $\mathrm{I}^{-}$ions in methanol- $N, N$-dimethylformamide mixtures. Thermochim Acta. 1990;170:189-95.

22. Piekarska A, Taniewska-Osińska S. Single-ion transfer enthalpies in methanol-acetonitrile mixtures at $298.15 \mathrm{~K}$ based on $\mathrm{Ph}_{4} \mathrm{P}^{+}=\mathrm{BPh}_{4}^{-}$assumption. Thermochim Acta. 1992;194:109-16.

23. Cox BG, Waghorne WE. Free energies, enthalpies and entropies of transfer of electrolytes from propylene carbonate to dimethylsulphoxide + propylene carbonate mixtures and from methanol to acetonitrile + methanol mixtures at $25{ }^{\circ} \mathrm{C}$. J Chem Soc Faraday Trans 1. 1984;80:1267-78.

24. Piekarski H, Kobierski W. Enthalpic pair interaction coefficients of NaI-non-electrolyte mixtures in DMF solution at $25^{\circ} \mathrm{C}$. Part 2. Application of the group additivity concept to interactions in DMF solution. Thermochim Acta. 1990;164:323-8.

25. Piekarski H, Waliszewski D. Enthalpic pair interaction coefficients in DMF solution. Part 3. Thermochemistry of NaI solutions in mixtures of $\mathrm{N}, \mathrm{N}$-dimethylformamide with urea, formamide, acetamide and $N, N$-dimethylacetamide at $298.15 \mathrm{~K}$. J Therm Anal. 1996;47:1639-47.

26. de Visser C, Somsen G. Enthalpies of tetra-n-butylammonium bromide in binary mixtures of water, formamide, $N$-methylformamide, and $N, N$-dimethylformamide. J Solution Chem. 1974;3: $847-55$.
27. Piekarski H, Waliszewski D, Romanowski S. Interactions of urea with some non-electrolytes in DMF solution. Calorimetric measurements and quantum-mechanical calculations. J Molec Liquids. 2000;88:43-54.

28. Piekarski H, Kubalczyk K. Thermochemical properties of ions in acetonitrile-organic cosolvent mixtures at $298.15 \mathrm{~K}$. J Molec Liquids. 2005;121:35-40.

29. Piekarski H, Kubalczyk K. Thermochemical examination of NaI, $\mathrm{NaBPh}_{4}$ and Ph4PI in the mixtures of acetonitrile with $\mathrm{N}, \mathrm{N}$ dimethylformamide and dimethylsulfoxide at $298.15 \mathrm{~K}$ : properties of acetonitrile-containing mixtures as solvents for electrolytes. J Chem Eng Data. 2010;55:1945-50.

30. Piekarski H, Kubalczyk K. Single ionic volumes in acetonitrileorganic cosolvent mixtures at 298.15 K. Z Phys Chem. 2004;218: 679-98.

31. Piekarski H, Kubalczyk K, Wasiak M. Volumes, heat capacities and compressibilities of the mixtures of acetonitrile with $\mathrm{N}, \mathrm{N}$ dimethylacetamide and propylene carbonate. J Chem Eng Data. 2010;55:5435-40.

32. Piekarski H, Piekarska A, Kubalczyk K. Volumes, heat capacities, and compressibilities of the mixtures of acetonitrile and 2-methoxyethanol. J Chem Thermodyna. 2011;43:1375-80. 